\title{
Quantum tomography of an electron
}

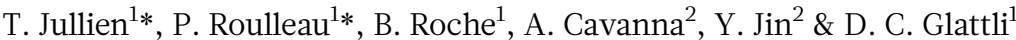

The complete knowledge of a quantum state allows the prediction of the probability of all possible measurement outcomes, a crucial step in quantum mechanics. It can be provided by tomographic methods ${ }^{1}$ which have been applied to atomic ${ }^{2,3}$, molecular $^{4}, \operatorname{spin}^{5,6}$ and photonic states. For optical ${ }^{7-9}$ or microwave ${ }^{10-13}$ photons, standard tomography is obtained by mixing the unknown state with a large-amplitude coherent photon field. However, for fermions such as electrons in condensed matter, this approach is not applicable because fermionic fields are limited to small amplitudes (at most one particle per state), and so far no determination of an electron wavefunction has been made. Recent proposals involving quantum conductors suggest that the wavefunction can be obtained by measuring the time-dependent current of electronic wave interferometers ${ }^{14}$ or the current noise of electronic Hanbury-Brown/Twiss interferometers ${ }^{15-17}$. Here we show that such measurements are possible despite the extreme noise sensitivity required, and present the reconstructed wavefunction quasiprobability, or Wigner distribution function ${ }^{17}$, of single electrons injected into a ballistic conductor. Many identical electrons are prepared in well-controlled quantum states called levitons ${ }^{18}$ by repeatedly applying Lorentzian voltage pulses to a contact on the conductor ${ }^{19-21}$. After passing through an electron beam splitter, the levitons are mixed with a weak-amplitude fermionic field formed by a coherent superposition of electron-hole pairs generated by a small alternating current with a frequency that is a multiple of the voltage pulse frequency ${ }^{16}$. Antibunching of the electrons and holes with the levitons at the beam splitter changes the leviton partition statistics, and the noise variations provide the energy density matrix elements of the levitons. This demonstration of quantum tomography makes the developing field of electron quantum optics with ballistic conductors a new test-bed for quantum information with fermions $s^{20,22-24}$. These results may find direct application in probing the entanglement of electron flying quantum bits ${ }^{25}$, electron decoherence ${ }^{17}$ and electron interactions. They could also be applied to cold fermionic (or spin1/2) atoms ${ }^{26}$.

A quantum state $\psi$ contains all the information about a particle or a system. Disregarding spin for simplicity, a practical representation is given by the product of the wavefunction $\varphi$ which contains the spatial information and an occupation state in a Fock space representation. Although the determination of the latter requires similar experimental resources for a fermion and for a single boson, the complete determination of the wavefunction by tomographic methods is fundamentally more demanding for fermions than for bosons. To emphasize this let us consider a single particle propagating in a single spatial mode (an electron in an effectively one-dimensional ballistic conductor or a photon in an optical medium). In the space and time domain representation, the electron quantum state at position $x$ and time $t$ is $\varphi\left(t-x / v_{\mathrm{F}}\right)|f\rangle$, where $v_{\mathrm{F}}$ is the velocity, $\varphi$ is the wavefunction and $|f\rangle$ is the fermionic occupation state, $|1\rangle$ or $|0\rangle$. Similarly, a photonic state is characterized by $E_{0} u(t-x / c)|b\rangle$, where $E_{0}$ is the electric field amplitude of a single photon, $u$ is the spatial mode which plays the role of $\varphi,|b\rangle$ is, for example, a number state or a Glauber coherent state, and $c$ is the speed of light. In quantum optics, the determination of the mode $u$ can be done by mixing with a coherent field (local oscillator) with amplitude $\sqrt{\bar{N}_{\mathrm{LO}}} E_{0} u(t-x / c)$, where $\bar{N}_{\mathrm{LO}}$ is the mean photon number. Then a classical measurement of $u$ can be made because the fundamental quantum measurement uncertainty $\sim 1 / \sqrt{\bar{N}_{\mathrm{LO}}}$ vanishes for large $\sqrt{\bar{N}_{\mathrm{LO}}}$ Only the determination of the bosonic state $|b\rangle$ requires purely quantum tomography. For fermions, however, the state occupation being limited to 1, no classical amplitude level can be reached and a quantum tomography is necessary for both the wavefunction $\varphi$ and the fermion state $|f\rangle$. In the present work, we prepare many electrons, each in the known fermionic state $|f\rangle=|1\rangle$. We show that, although extremely demanding, a quantum tomography of the wavefunction of a time-resolved single electron is possible using shot noise, and we determine the first-order coherence $\tilde{\varphi}^{*}\left(\varepsilon^{\prime}\right) \tilde{\varphi}(\varepsilon)$ in the energy representation (where $\tilde{\varphi}(\varepsilon)$ is the Fourier transform of $\varphi(t)$ and the asterisk denotes complex conjugation).

We first explain how we prepare the single-electron state in a quantum conductor and then how the quantum tomography is performed. The quantum conductor is a quantum point contact (QPC) placed at low temperature (electron temperature, $T_{\mathrm{e}} \approx 35 \mathrm{mK}$ ). It is formed in a high-mobility two-dimensional electron gas using split gates deposited on top of a GaAs/GaAlAs heterojunction (Fig. 1a and Supplementary Information). Applying negative voltage $V_{\mathrm{G}}$ to the gates creates a constriction transmitting a limited number of electronic modes. Here we select a single mode whose transmission $D\left(V_{\mathrm{G}}\right)$ is obtained by measuring the conductance $G=\left(2 e^{2} / h\right) D$, where $e$ is the electron charge and $h$ is Planck's constant. The on-demand injection of charges into the conductor is obtained by applying a voltage pulse $V_{\mathrm{L}}(t)$ on one of the ohmic contacts adjacent to the QPC, say the left. If the voltage flux satisfies $e \int_{-\infty}^{+\infty} V_{\mathrm{L}}(t) \mathrm{d} t=h$ then the charge is unity. Moreover, a Lorentzian pulse $V(t)=2 \hbar w / e\left(t^{2}+w^{2}\right)$, where $\hbar=h / 2 \pi$ and $2 w$ is the pulse width at mid height, creates a clean single-electron state ${ }^{19}$ called a leviton ${ }^{18}$ with an energy just greater than the Fermi energy of the conductor. Remarkably, although the leviton originates from a collective displacement of the Fermi sea towards positive energy, it can be considered a single-particle state ${ }^{20,21}$ disentangled from the Fermi sea ${ }^{21}$. A wavefunction representation of the leviton in the energy domain is $\tilde{\varphi}(\varepsilon)=\sqrt{2 w / \hbar} \theta(\varepsilon) \mathrm{e}^{-\varepsilon w / \hbar}$ where $\theta$ is the Heaviside step function. Experimentally, periodic trains of levitons have been generated ${ }^{18}$. When partitioned by a QPC ${ }^{18}$ they generate a current shot noise corresponding to electrons prepared in the occupation state $|1\rangle$. Using shot-noise spectroscopy and electron Hong-Ou-Mandel noise correlation techniques, their wavefunction probability in the energy and time domain was found to be consistent with expectations. Thus, levitons provide a clean way of testing wavefunction quantum tomography in a quantum conductor.

We now discuss how to probe the first-order coherence from which the full wavefunction can be extracted. A first approach could be wave interferometry. In quantum optics, this gives $G_{1}\left(t-t^{\prime}\right)$, which is the firstorder coherence $\left\langle\psi^{+}\left(t^{\prime}\right) \psi(t)\right\rangle$ averaged over the mean time $\bar{t}=\left(t+t^{\prime}\right) / 2$ (here $\psi^{+}$is the Hermitian conjugate of $\psi$ ). The information is incomplete, however, and other approaches must be used ${ }^{27}$. Wave interferometry is more promising with electrons: measuring the time-dependent current of an electronic Mach-Zehnder interferometer has been proposed $^{14}$ as an elegant way to determine the full coherence $\left\langle\psi^{+}\left(t^{\prime}\right) \psi(t)\right\rangle$,

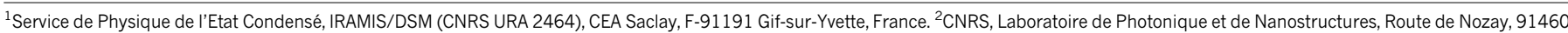
Marcoussis, France.

*These authors contributed equally to this work. 

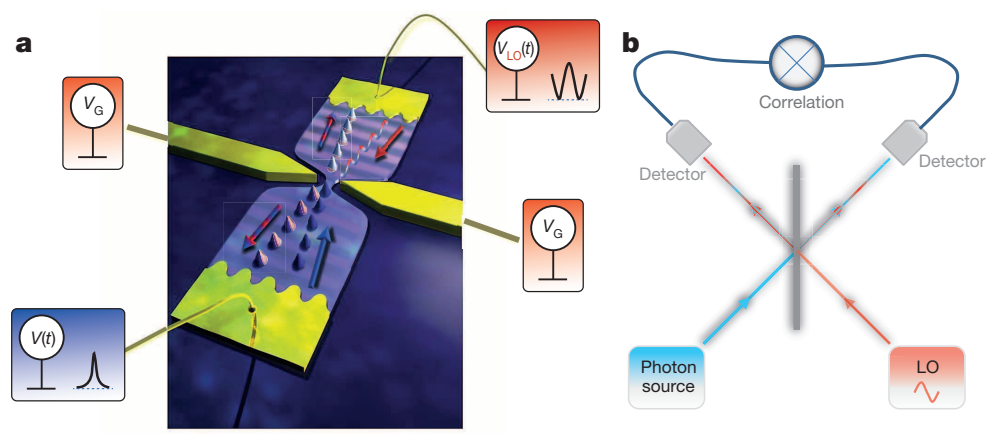

c

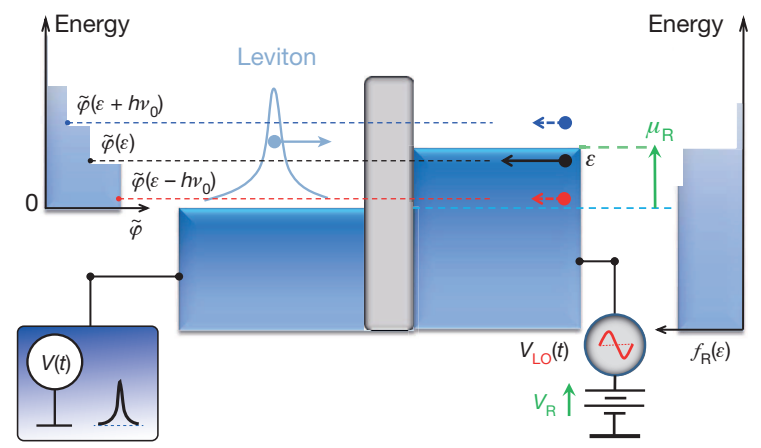

Figure 1 Schematics of quantum wave tomography. a, Experiment principle. Periodic Lorentzian voltage pulses $V(t)$ applied to the left contact of a two-dimensional electron gas inject unit charge pulses called levitons which are partitioned by an electronic beam splitter called a quantum point contact (QPC). The split-gate voltage $V_{\mathrm{G}}$ controls the transmission $D$ of the onedimensional electronic mode formed at the QPC. A d.c. voltage $V_{\mathrm{R}}$ (not shown) and a weak a.c. voltage $V_{\mathrm{LO}}(t)=\left(k \eta_{\mathrm{LO}} h v_{0} / e\right) \cos \left(2 \pi k v_{0}(t-\tau)\right)$ are applied to the right contact The latter generates a small flux of electrons and holes (red-blue wavy line) which interferes with the incoming levitons (blue bumps) and is analogous to the local oscillator used in quantum optics. The red and blue arrows indicate the directions of electron-hole and leviton excitation propagation, respectively. Bicolour symbols indicate the outgoing states made of levitons mixed with the small flux of electron and hole excitations. Measuring the low-frequency current shot noise while varying $V_{\mathrm{R}}$ for different harmonics $(k=0,1$ and 2) provides a tomographic measurement of the leviton energy density matrix. b, Optical analogue. The local oscillator (LO) emits a monochromatic photon field, here of high amplitude, which interferes in the beam splitter with photons emitted by the source to be analysed. The photons detected provide a tomographic measurement of the photonic state.

but so far no such experiments have been done. Another approach is performing shot-noise measurements. This is the approach followed in the present experimental work. Photon shot noise has been exploited using adaptive homodyne tomography ${ }^{27}$ to infer the unknown spectrotemporal properties of a single photon mode $u$. A large local oscillator field is mixed with the single photon in a beam splitter and its temporal shape is tuned to maximize the output noise, providing a classical copy of the unknown mode (Fig. 1b). For electrons, a large fermion field cannot be used but the principle of homodyne mixing can be kept. A recent proposal is to reverse the amplitude hierarchy ${ }^{16}$. Because it is the single-electron source corresponding to the maximum fermionic amplitude (as $|f\rangle=|1\rangle)$, this is the local oscillator fermionic probe source whose amplitude has to be weak so that simple information can be provided by the noise variation at the beam-splitter output when mixing particles coming from the two sources. For simplicity, let us consider zero temperature. When a flux-quantized Lorentzian voltage pulse is applied to the left contact, a leviton is sent towards the QPC beam splitter. Repeating the experiment and recording the charge at the right contact gives the mean charge $\langle Q\rangle=D e$ and the charge partition noise $\left\langle\Delta Q^{2}\right\rangle=D(1-D) e^{2}$. Now let us apply a d.c. voltage $V_{\mathrm{R}}$ to the right contact, rising the electrochemical potential by $e V_{\mathrm{R}}$. The part of the leviton partition noise proportional to $\int_{0}^{e V_{\mathrm{R}}} \tilde{\varphi}(\varepsilon)^{*} \tilde{\varphi}(\varepsilon) \mathrm{d} \varepsilon$, corresponding to the energy range $\varepsilon \in\left[0, e V_{\mathrm{R}}\right]$, is

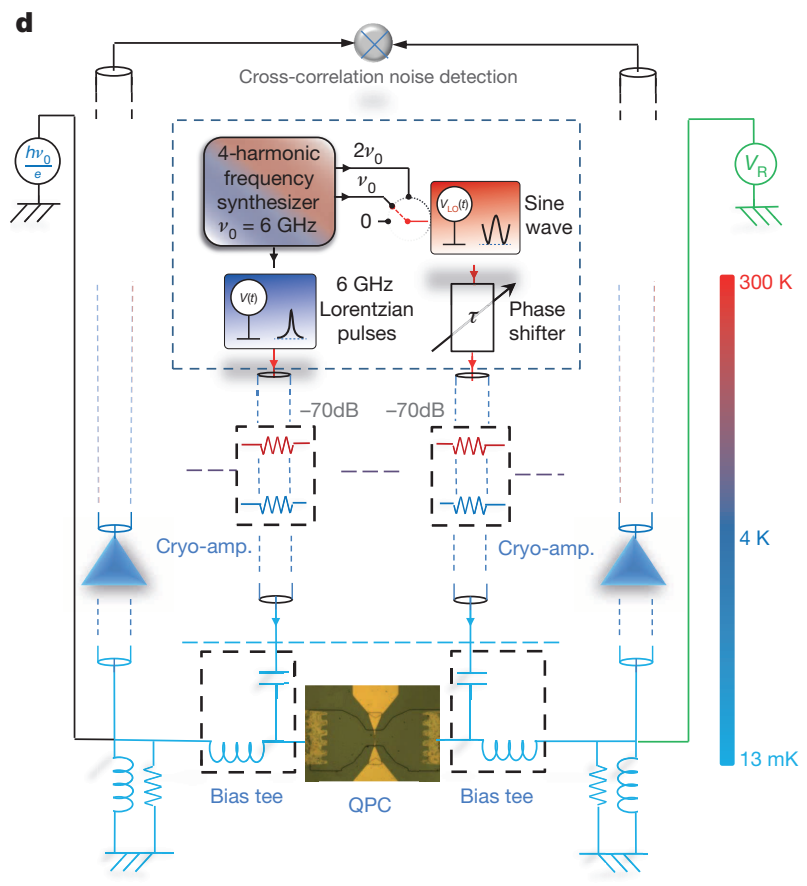

c, Experiment principle in energy representation. All quantities are step functions of the energy with energy scale $h v_{0}$. Left: the energy dependence of the wavefunction of periodic levitons emitted from the left contact. Right: the energy distribution of the right reservoir when the voltage $V_{\mathrm{R}}+V_{\mathrm{LO}}(t)$ is applied. For small $\eta_{\mathrm{LO}}$, an electron emitted with energy $\varepsilon$ arrives at the QPC in a superposition of states of energy $\varepsilon \pm k h v_{0}$. Its interference with the levitons changes the leviton partition noise by a quantity proportional to $\eta_{\mathrm{LO}}\left(\tilde{\varphi}(\varepsilon) \tilde{\varphi}\left(\varepsilon+k h v_{0}\right)-\tilde{\varphi}\left(\varepsilon-k h v_{0}\right) \tilde{\varphi}(\varepsilon)\right)$, giving information on the non-diagonal energy density matrix. $f_{\mathrm{R}}=|\varphi(\varepsilon)|^{2}$. d, The $6 \mathrm{GHz}$ periodic Lorentzian voltage pulses are obtained from a frequency synthesizer by combining four harmonics with appropriate phases and amplitudes and sent to the left ohmic contact of the sample via $40 \mathrm{GHz}$ d.c. transmission lines. To generate $V_{\text {LO }}$ harmonics 1 and 2 are derived from the synthesizer, attenuated and sent to the right contact. A time delay is added using a phase shifter. Bias tees separate the high-frequency components from the d.c. voltage bias and the detected low-frequency current noise. The latter is converted into voltage fluctuations. A computer performs fast Fourier transform cross-correlations after cryogenic amplification and fast acquisition. Appropriate d.c. voltage biases are applied to the left and right contacts.

replaced by the partition noise of the right electrons reduced by the factor $1-\int_{0}^{e V_{\mathrm{R}}} \tilde{\varphi}(\varepsilon)^{*} \tilde{\varphi}(\varepsilon) \mathrm{d} \varepsilon$ because of antibunching. Differentiating the noise with respect to $V_{\mathrm{R}}$ provides the diagonal part of the energy density matrix of the leviton $\left|\tilde{\varphi}\left(\varepsilon=e V_{\mathrm{R}}\right)\right|^{2}$. Remarkably, very little modification is necessary to access the non-diagonal part $\left\langle\psi^{+}\left(\varepsilon^{\prime} \neq \varepsilon\right) \psi(\varepsilon)\right\rangle$. Let us superimpose on the right d.c. voltage the a.c. voltage $V_{\mathrm{LO}}(t)=$ $\eta_{\mathrm{LO}}(h v / e) \cos (2 \pi v(t-\tau))$ with small amplitude $\eta_{\mathrm{LO}} \ll 1$, frequency $v$ and time delay $\tau$ referred to the leviton emission time. This generates at the QPC a weak fermionic field made of electrons in a superposition of states with their initial energies shifted by $\pm h v$ and with probability amplitudes $\eta_{\mathrm{LO}} \mathrm{e}^{i 2 \pi v \tau} / 2$ and $-\eta_{\mathrm{LO}} \mathrm{e}^{-i 2 \pi v \tau} / 2$, respectively, the probability of keeping the initial energy being $1-\eta_{\mathrm{LO}}{ }^{2} \approx 1$ (Fig. 1c). Their mixing with the levitons at the beam splitter gives the antibunching probability amplitude

$$
\begin{aligned}
a\left(\eta_{\mathrm{LO}}, \varepsilon\right)= & \tilde{\varphi}^{*}(\varepsilon)\left(1-\eta_{\mathrm{LO}}{ }^{2}\right)+\frac{1}{2} \tilde{\varphi}^{*}(\varepsilon+h v) \eta_{\mathrm{LO}} \mathrm{e}^{i 2 \pi v \tau} \\
& -\frac{1}{2} \tilde{\varphi}^{*}(\varepsilon-h v) \eta_{\mathrm{LO}} \mathrm{e}^{-i 2 \pi v \tau}
\end{aligned}
$$

Differentiating the noise with respect to $e V_{\mathrm{R}}$, and keeping only the a.c. contribution, gives a noise variation proportional to 


$$
\begin{aligned}
\eta_{\mathrm{LO}} \operatorname{Re}\left[\tilde{\varphi}^{*}\left(e V_{\mathrm{R}}\right) \tilde{\varphi}\left(e V_{\mathrm{R}}+h v\right) \mathrm{e}^{-i 2 \pi v \tau}\right. \\
\left.-\tilde{\varphi}^{*}\left(e V_{\mathrm{R}}\right) \tilde{\varphi}\left(e V_{\mathrm{R}}-h v\right) \mathrm{e}^{i 2 \pi v \tau}\right]
\end{aligned}
$$

Fully quantum tomography can then be done by varying the voltage frequency and time delay $\tau$, providing the real and imaginary parts of the first-order coherence ${ }^{16}$. Because the leviton wavefunction in the energy domain is real, we get

$$
\begin{array}{r}
\frac{\mathrm{d}\left\langle\Delta Q^{2}\right\rangle}{\mathrm{d} V_{\mathrm{R}}} \propto \eta_{\mathrm{LO}} \cos (2 \pi v \tau) \\
{\left[\tilde{\varphi}^{*}\left(e V_{\mathrm{R}}\right) \tilde{\varphi}\left(e V_{\mathrm{R}}+h v\right)\right.} \\
\left.-\tilde{\varphi}^{*}\left(e V_{\mathrm{R}}\right) \tilde{\varphi}\left(e V_{\mathrm{R}}-h v\right)\right]
\end{array}
$$

Because only positive energy is concerned, equation (1) gives $\tilde{\varphi}\left(e V_{\mathrm{R}}\right) \tilde{\varphi}$ $\left(e V_{\mathrm{R}}+h v\right)$ for $0 \leq e V_{\mathrm{R}}<h v, \tilde{\varphi}\left(e V_{\mathrm{R}}\right) \tilde{\varphi}\left(e V_{\mathrm{R}}+h v\right)-\tilde{\varphi}\left(e V_{\mathrm{R}}-h v\right) \tilde{\varphi}\left(e V_{\mathrm{R}}\right)$ for $h v \leq e V_{\mathrm{R}}<2 h v$ and so on, providing a full determination of the energy density matrix.

In our experiment, single-electron levitons are injected at a frequency $v_{0}$ by applying periodic Lorentzian voltage pulses from the left contact. The full-width at mid-height is $2 w=30$ ps and the period is $T=v_{0}{ }^{-1}=166 \mathrm{ps}$. To extract the diagonal and off-diagonal parts of the energy density matrix, we follow the experimental approach discussed above with current noise measured instead of charge fluctuations. With $V_{\mathrm{R}}=0$ and in the absence of a.c. voltage, the partitioning of levitons generates the low-frequency current noise spectral density $S_{I}^{0}(0)=4 v_{0}$ $\left\langle\Delta Q^{2}\right\rangle=4 v_{0} e^{2} D(1-D)$ where double counting of the mode from spin degeneracy is included. Because of the periodicity, $\left\langle\psi^{+}\left(\varepsilon^{\prime}\right) \psi(\varepsilon)\right\rangle=$ $\sum_{k} \delta\left(\varepsilon^{\prime}-\varepsilon-k h v_{0}\right) \tilde{\varphi}^{*}\left(\varepsilon+k h v_{0}\right) \tilde{\varphi}(\varepsilon)$, where $\delta$ is the Dirac delta function and $k$ is an integer. Only energies separated by multiple of $h v_{0}$ contribute to off-diagonal terms, and the frequency of $V_{\mathrm{LO}}(t)$ must be $v= \pm k v_{0}$. Because high harmonics give too small a signal, measurements are restricted to $k=0,1$ and 2 .

The diagonal part $(k=0)$ is obtained by measuring the difference between the partition noise of single-electron levitons injected at frequency $v_{0}$, using the periodic Lorentzian voltage $V_{\mathrm{L}}(t)$, and that of continuously injected electrons provided by a constant voltage equal to the mean value of the voltage pulse $\bar{V}_{L}(t)=h v_{0} / e$. The zero-temperature noise difference is ${ }^{18,28}$

$\Delta S_{I}^{k=0}\left(\mu_{\mathrm{R}}\right)=\frac{S_{I}^{0}}{h v_{0}}\left(\int_{0}^{\mu_{\mathrm{R}}}\left(1-\tilde{\varphi}(\varepsilon)^{2}\right) \mathrm{d} \varepsilon-\int_{\mu_{\mathrm{R}}}^{\infty} \tilde{\varphi}(\varepsilon)^{2} \mathrm{~d} \varepsilon-\left|\mu_{\mathrm{R}}-h v_{0}\right|\right)$

On the right-hand side, the first term represents the noise of right electrons emitted at energy below $\mu_{\mathrm{R}}=e V_{\mathrm{R}}$, whose shot noise is reduced by antibunching with the leviton. The second term is the partition noise of levitons for energy above $\mu_{\mathrm{R}}$. It is followed by the subtracted d.c. shot noise. Because $\varphi(\varepsilon)$ varies by steps in $h v_{0}$ intervals, equation (2) displays linear variation by parts. Introducing the notation $\tilde{\varphi}(\varepsilon)^{2}=\rho_{l, l}$ for $l h v_{0}<\varepsilon<(l+1) h v_{0}$, this yields 0 for $\mu_{\mathrm{R}}<0,\left(2\left(1-\rho_{0,0}\right) \mu_{\mathrm{R}}+h v_{0}-\mu_{\mathrm{R}}\right) /$ $h v_{0}$ for $0<\mu_{\mathrm{R}}<h v_{0}, 2\left(1-\rho_{0,0}\right)-2 \rho_{1,1}\left(\mu_{\mathrm{R}}-h v_{0}\right) / h v_{0}$ for $h v_{0}<\mu_{\mathrm{R}}$ $<2 h v_{0}$, and so on. The finite electron temperature $T_{\mathrm{e}} \approx 35 \mathrm{mK} \approx$ $0.12 h v_{0} / k_{\mathrm{B}}$ smoothens the singularities separating linear variations. A finite-temperature expression, obtained in a Floquet scattering approach ${ }^{29,30}$, is given in Supplementary Information. Figure 2a shows measurements of $\Delta S_{I}^{k=0}$ versus $\mu_{R}$ for levitons (open blue circles). From the data we extract $\rho_{l, l}$ directly from a fit including the small-temperature effects. The solid red curve corresponds to the best four-parameter fit, from which the four first $\rho_{l, l}$ (that is, $l=0$ to 3 ) are extracted (Supplementary Information). Self-heating including a small temperature increase $(35 \mathrm{mK}$ for $V_{\mathrm{R}} \approx 0$ to $47 \mathrm{mK}$ for $V_{\mathrm{R}} \approx 3 h v_{0} / e$ ) has been included. The $\rho_{l, l}$ are plotted in red in Fig. 2b. Comparison with theoretical values based on a Floquet scattering modelling (in black) is good within the experimental uncertainties.

For the measurement of the off-diagonal part $(k \neq 0)$, we measure the noise difference when switching on and off the amplitude of
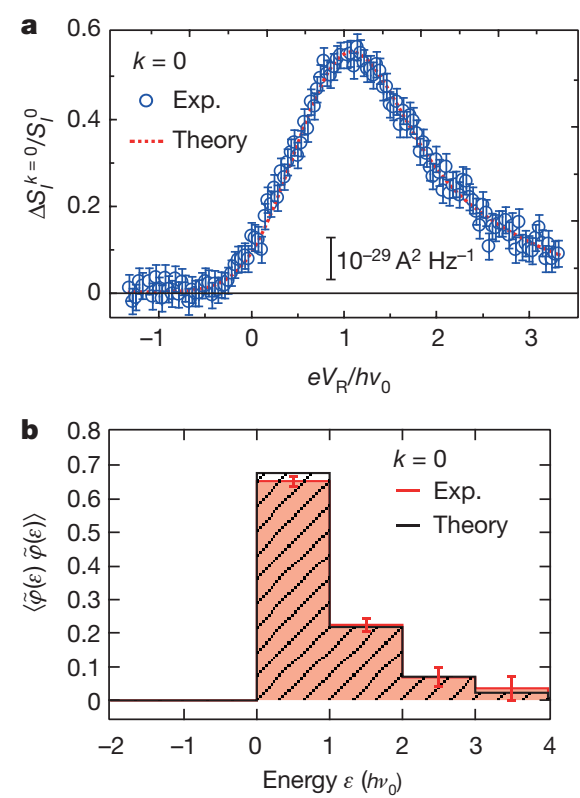

Figure $2 \mid$ Measurement of the diagonal part of the energy density matrix. a, The data (blue circles) display the shot noise measured when applying a d.c. voltage $h v_{0} / e$, minus the shot noise measured when sending single-charge levitons at frequency $v_{0}$. The data are plotted versus the d.c. voltage $V_{\mathrm{R}}$ applied on the opposite contact. The red curve represents the best fit to equation (2), including finite temperature, from which the parameters $\rho_{l, l}$ are extracted. The temperature $T_{\mathrm{e}}$ is $\sim 35 \mathrm{mK}$ at $V_{\mathrm{R}}=0$ and $\sim 48 \mathrm{mK}$ at $V_{\mathrm{R}}=3 h v_{0} / e$. The transmission is $D=0.19$ and $S_{I}^{0}=9.48 \times 10^{-29} \mathrm{~A}^{2} \mathrm{~Hz}^{-1}$. Error bars, s.e.m. calculated from the cross-correlation noise spectrum standard deviation in a $800 \mathrm{kHz}$ bandwidth and after $n \approx 100,000$ acquisitions. $\mathbf{b}$, Diagonal part of the energy density matrix versus energy using the $\rho_{l, l}$ extracted from the fit. Comparison with theory for levitons is in black. Error bars, s.e. calculated from the best fit.

$V_{\mathrm{LO}}(t)=\eta_{\mathrm{LO}}(h v / e) \cos (2 \pi v(t-\tau))$ superimposed on the right d.c. voltage $V_{\mathrm{R}}$ and synchronized with the Lorentzian pulses. The zerotemperature shot noise difference is

$$
\begin{aligned}
\Delta S_{I}^{k}\left(\mu_{\mathrm{R}}\right)= & \frac{S_{I}^{0}}{h v_{0}} 2 k \eta_{\mathrm{LO}} \cos \left(2 \pi k v_{0} \tau\right) \\
& \times \int_{0}^{\mu_{\mathrm{R}}}\left(\tilde{\varphi}(\varepsilon) \tilde{\varphi}\left(\varepsilon+k h v_{0}\right)-\tilde{\varphi}(\varepsilon) \tilde{\varphi}\left(\varepsilon-k h v_{0}\right)\right) \mathrm{d} \varepsilon
\end{aligned}
$$

This expression also varies linearly by parts because $\tilde{\varphi}(\varepsilon) \tilde{\varphi}\left(\varepsilon+k h v_{0}\right)$ $=\rho_{l, l+k}$ for $l h v_{0}<\varepsilon<(l+1) h v_{0}$ is a step function of the energy. For example, for $k=1$ the integral in equation (3) is 0 for $\mu_{\mathrm{R}}<0, \rho_{0,1} \mu_{\mathrm{R}} /$ $h v_{0}$ for $0<\mu_{\mathrm{R}}<h v_{0}, \rho_{0,1}-\rho_{1,2}\left(\mu_{\mathrm{R}}-h v_{0}\right) / h v_{0}$ for $h v_{0}<\mu_{\mathrm{R}}<2 h v_{0}$, and so on. Finite-temperature expressions are given in Supplementary Information.

Figure $3 \mathrm{a}, \mathrm{b}$ shows measurements of $\Delta S_{I}^{k}$ versus $\tau$ for $k=1, \eta_{\mathrm{LO}}$ $=0.095$ and $k=2, \eta_{\mathrm{LO}}=0.0425$ at fixed $\mu_{\mathrm{R}} \approx h v_{0}$. Clear oscillations are observed. The delay period in Fig. $3 \mathrm{~b}$ is twice that observed in Fig. 3a. Before going further, we emphasize that observation of these oscillations demonstrates off-diagonal coherence in an electronic orbital state, here a leviton. No experiment has given direct evidence of off-diagonal coherence of electrons in a conductor yet. We now compare the offdiagonal terms extracted from the measurements with those predicted for a leviton. Figure $3 c, d$ shows the experimental variation of $\Delta S_{I}^{k}$ with $\mu_{\mathrm{R}}$ for $k=1$ and 2 (open blue circles), fixing $\tau=0$ to maximize the signal. In both plots, the solid black curves represent the theoretical signal expected for a leviton. The red dashed curves are the best fits, from which the off-diagonal terms $\rho_{l, l+k}$ can be extracted. For $k=2$ a four-parameter fit is used and $l=0$ to 3 , and for $k=1$ a five-parameter fit is used because a finite, but very small, hole excitation contribution 
a
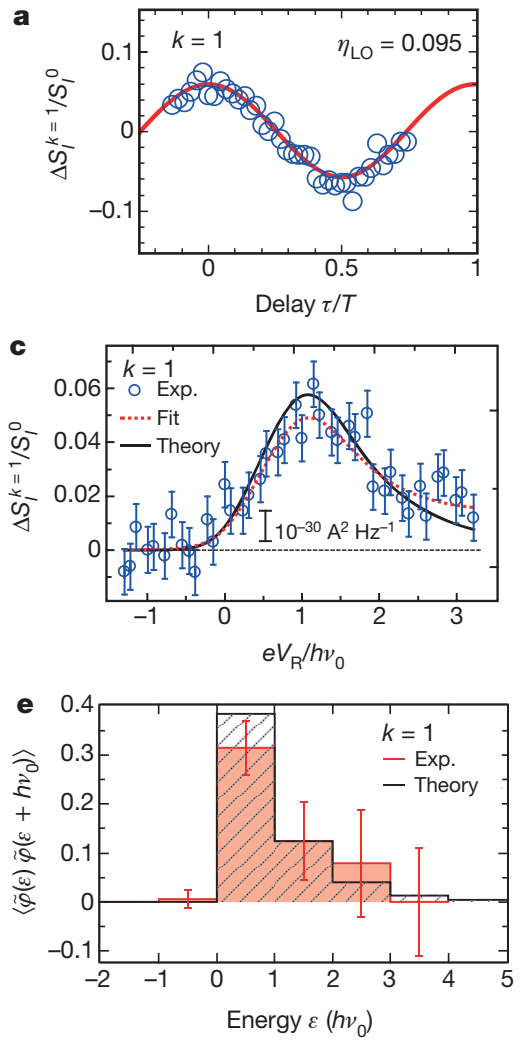

a

Experiment
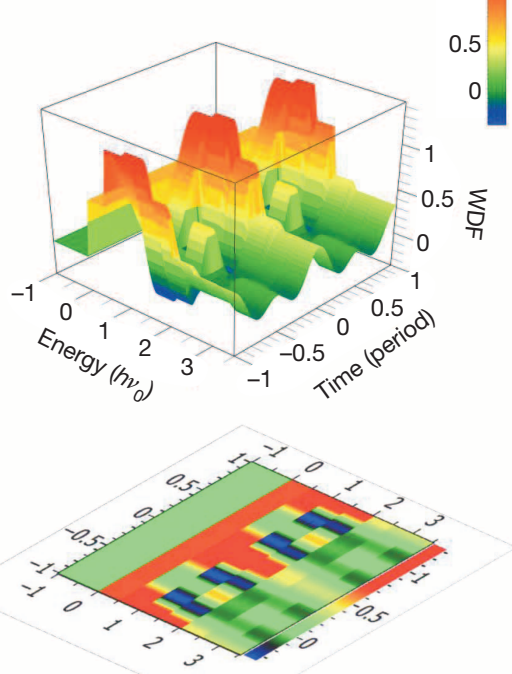

c

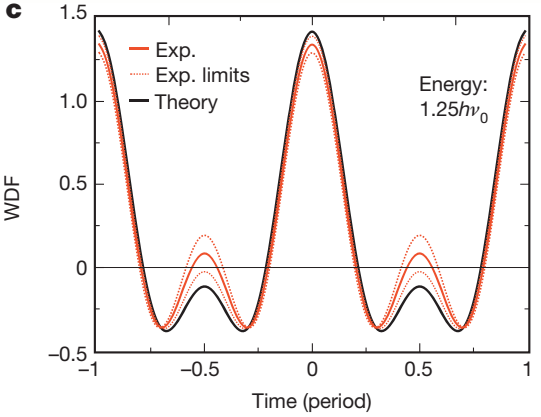

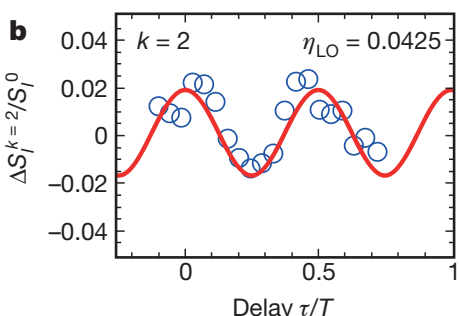
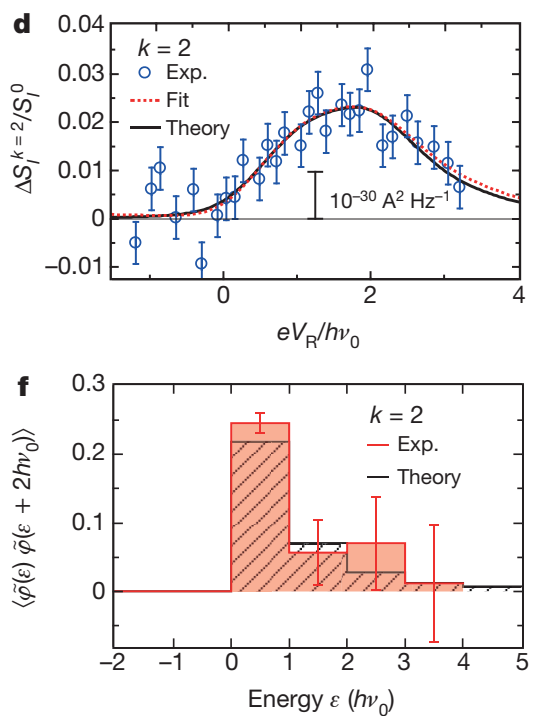

b
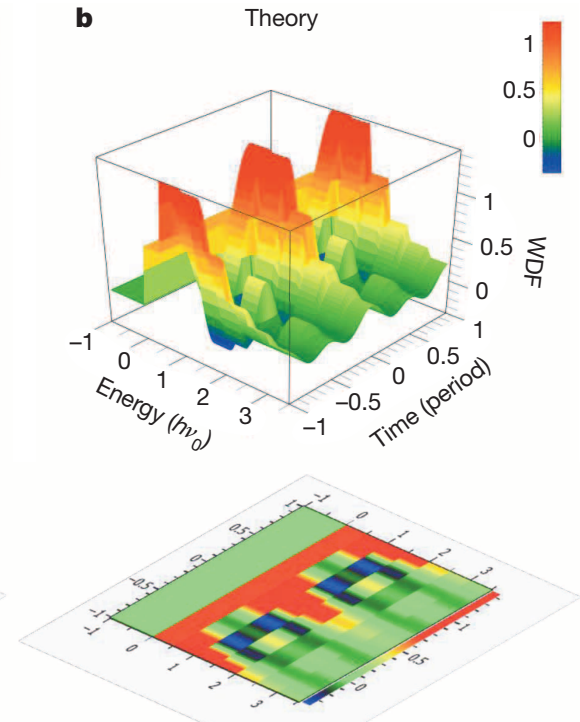

d

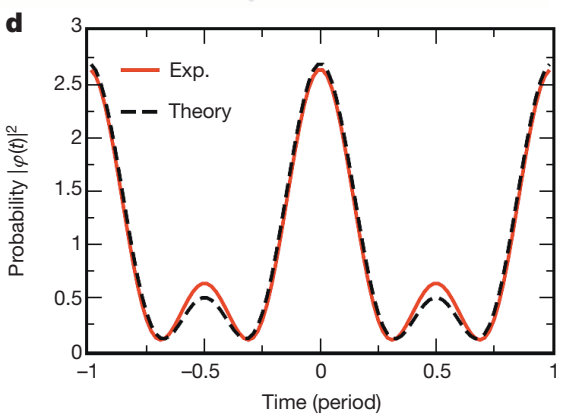

Figure $3 \mid$ Off-diagonal part of energy density matrix. a, b, Weak sine-wave voltages of frequencies and amplitudes $v_{0}$ and $\eta_{\mathrm{LO}}=0.095$ (a) and $2 v_{0}$ and $\eta_{\mathrm{LO}}=0.0425$ (b) superimposed on a fixed d.c. voltage $\mathrm{e} V_{\mathrm{R}}=h v_{0}$ applied on the right contact. The data (blue circles) show the noise difference obtained by switching on and off the sine-wave amplitude, plotted versus the time delay $\tau$ between the sine-wave and the periodic Lorentzian pulses. The oscillation of the noise with $\tau$ and the period doubled in $\mathbf{b}$ relative to $\mathbf{a}$ is the signature of off-diagonal coherence. The red curves are sinusoidal fits. c, d, Same shot noise difference measurements (blue circles) but plotted versus the d.c. right voltage with $\tau=0$, chosen to maximize the signal. $T_{\mathrm{e}}$ is $\sim 35 \mathrm{mK}$ at $V_{\mathrm{R}}=0$ and $\sim 48 \mathrm{mK}$ at $V_{\mathrm{R}}=3 h v_{0} / e$. The red curves are the best fit of equation (3) with $\rho_{l, l+\mathrm{k}}$ as free parameters, including finite-temperature effects, with $k=1$ (c) and $k=2(\mathbf{d})$. The black curves are comparison with theory. Error bars, s.e.m. calculated as in Fig. 2a but with $n$ four times larger. e, f, Plot of $\tilde{\varphi}(\varepsilon) \tilde{\varphi}\left(\varepsilon+h k v_{0}\right)$ (red) using the parameters $\rho_{l, l+\mathrm{k}}$ extracted from the best fits of $\mathbf{c}$ and $\mathbf{d}$ with $k=1$ (e) and $k=2(\mathbf{f})$, respectively. Expected values from theory are shown in black. Error bars, s.e. calculated from the best fit.
Figure $4 \mid$ Wigner function and leviton wavefunction in the time domain. a, The zerotemperature Wigner distribution function of the levitons is reconstructed from the $\rho_{l, l+\mathrm{k}}$ values obtained from tomography. The WDF is limited to the first two temporal harmonics and to the energy domain $\varepsilon<3.5 h v_{0}$. Negative parts are characteristic of a wavefunction which cannot be described by a classical probability distribution in the time and energy domains. $\mathbf{b}$, Theoretical WDF. The full WDF has been truncated to the first two harmonics and to the energy range probed experimentally, for better comparison. The two-dimensional colour plots are shown to better display the region of negative values of the WDF. c, Cut of the experimental and theoretical WDFs at energy $\varepsilon=1.25 h v_{0}$ (for energies $1.5 h v_{0}>\varepsilon>h v_{0}$ both the first and second harmonics contribute to the WDF). Using the experimental errors in Fig. 3e, f, we have generated the two red dashed curves between which the experimental WDF is expected to lie. Robust negative values of the WDF are found, a hallmark of interference effects. d, Projecting the WDF along the energy direction gives access to the temporal variation of the electron probability distribution of the leviton $|\varphi(t)|^{2}$. The experimental data are (red) are compared with the theoretical truncated WDF (black). 
$(l=-1)$ was found necessary to improve the fit. They are shown in Fig. 3e, f. The errors bars are obtained from the standard error of the fit. They are much larger than those in Fig. $2 b$ because the measured noise is about ten times smaller.

The noise oscillations with phase in Fig. $3 \mathrm{a}$, b and the noise variations with voltage in Fig. $3 c$, d are well reproduced by a model including only the $35-50 \mathrm{mK}$ thermal rounding. Supplementary Fig. 2 provides additional tomographic noise measurements performed on two-electron sine-wave pulses. Here again good agreement is found between data and theory. Deviation arising from decoherence effects due to electronelectron interaction is not expected here because the two-dimensional Landau quasi-particle lifetime is longer than the $(50 \mathrm{mK})$ thermal time for the typical energies probed here (Supplementary Information).

From the experimental values of $\tilde{\varphi}(\varepsilon) \tilde{\varphi}\left(\varepsilon+k h v_{0}\right)$ obtained above we can now reconstruct part of the zero-temperature Wigner distribution function ${ }^{17}(\mathrm{WDF})$ of the levitons: $W(\bar{t}, \varepsilon)=\int_{-\infty}^{+\infty}\left\langle\psi^{+}(\varepsilon+\delta / 2) \psi(\varepsilon-\right.$ $\delta / 2)\rangle \mathrm{e}^{-i \delta \bar{t} / \hbar} \mathrm{d} \delta$. The WDF gives the full information on the complex wavefunction. It has been calculated for a single Lorentzian pulse ${ }^{14}$ and for periodic pulses ${ }^{17}$. In the latter case, the WDF is also periodic. Its expression in terms of the quantities $\rho_{l, l+k}$ is given in Supplementary Information. Figure $4 \mathrm{a}$ and Fig. $4 \mathrm{~b}$ respectively give three-dimensional plots of the experimental and theoretical WDFs. Because the experimental WDF is limited to the first two harmonics and the energy range $-h v_{0} \leq \varepsilon<3.5 h v_{0}$, the theoretical WDF is truncated accordingly for comparison. To better show the agreement between experiment and theory, we provide in Fig. $4 \mathrm{c}$ a cut of the WDF at energy $\varepsilon=1.25 h v_{0}$ (red, experiment; black, theory). Using the experimental errors of $\rho_{l, l+k}$ $(l=0)$ in Fig. $3 \mathrm{e}, \mathrm{f}$, we have generated the two red dashed curves in Fig. $4 \mathrm{c}$ between which the experimental WDF is expected to be located. For reduced times $t / T$ around $\sim 0.3$ and 0.7 we clearly find robust regions of negative WDF, a hallmark of a quantum state.

Finally, as a particular application of the WDF, the integration over energy $\varepsilon$ gives the probability $|\varphi(t)|^{2}$. Its experimental (red solid curve) and theoretical (black dashed curve) variations with time are given in Fig. $4 \mathrm{~d}$. The excellent agreement validates our experimental determination of the WDF from the tomographic noise procedure of ref. 16. This provides better information on the temporal shape of the wavefunction than the convolution $|\langle\varphi(t) \mid \varphi(t+\tau)\rangle|^{2}$ given by the electronic Hong$\mathrm{Ou}-\mathrm{Mandel}$ method used for levitons in ref. 18 or for single electrons in ref. 31 .

Using the known leviton state, we have demonstrated that the quantum wave tomography of itinerant electrons can be done experimentally using shot noise. Probing the off-diagonal energy density matrix opens the possibility of studying more complex electron quantum states such as entangled electron quantum bits in ballistic conductors or complex electron quantum states built by interaction. Examples of the latter include Kondo electronic states, fractional quantum Hall quasi-particles or electron Andreev pairs built by superconducting proximity effects.

\section{Received 2 June; accepted 29 August 2014.}

1. Vogel, K. \& Risken, H. Determination of quasiprobability distributions in terms of probability distributions for the rotated quadrature phase. Phys. Rev. A 40, 2847-2849 (1989).

2. Leibfried, D. etal. Experimental determination of the motional quantum state of a trapped atom. Phys. Rev. Lett. 77, 4281-4285 (1996).

3. Kurtsiefer, C., Pfau, T. \& Mlynek, J. Measurement of the Wigner function of an ensemble of helium atoms. Nature 386, 150-153 (1997)

4. Dunn, T. J., Walmsley, I. A. \& Mukamel, S. Experimental determination of the quantum-mechanical state of a molecular vibrational mode using fluorescence tomography. Phys. Rev. Lett. 74, 884-887 (1995).
5. Shulman, M. D. et al. Demonstration of entanglement of electrostatically coupled singlet-triplet qubits. Science 336, 202-205 (2012).

6. Medford, J. et al. Self-consistent measurement and state tomography of an exchange-only spin qubit. Nature Nanotechnol. 8, 654-659 (2013).

7. Smithey, D. T., Beck, M., Raymer, M. G. \& Faridani, A. Measurement of the Wigner distribution and the density matrix of a light mode using optical homodyne tomography: application to squeezed states and the vacuum. Phys. Rev. Lett. 70, 1244-1247 (1993)

8. Breitenbach, G. Schiller, S.\& Mlynek, J. Measurement of the quantum states of squeezed light. Nature 387, 471-475 (1997).

9. Lvovsky, A. I. et al. Quantum state reconstruction of the single-photon Fock state. Phys. Rev. Lett. 87, 050402 (2001)

10. Bertet, P. et al. Direct measurement of the Wigner function of a one-photon Fock state in a cavity. Phys. Rev. Lett. 89, 200402 (2002).

11. Houck, A. A. et al. Generating single microwave photons in a circuit. Nature $\mathbf{4 4 9}$ 328-331 (2007).

12. Hofheinz, M. et al. Synthesizing arbitrary quantum states in a superconducting resonator. Nature 459, 546-549 (2009).

13. Eichler, C. et al. Experimental tomographic state reconstruction of itinerant microwave photons. Phys. Rev. Lett. 106, 220503 (2011).

14. Haack, G., Moskalets, M. \& Büttiker, M. Glauber coherence of single-electron sources. Phys. Rev. B 87, 201302(R) (2013).

15. Samuelsson, P. \& Büttiker, M. Quantum sate tomography with quantum shot noise. Phys. Rev. B 73, 041305(R) (2006).

16. Grenier, C. et al. Single-electron quantum tomography in quantum Hall edge channels. New J. Phys. 13, 093007 (2011)

17. Ferraro, D. et al. Wigner function approach to single electron coherence in quantum Hall edge channels. Phys. Rev. B 88, 205303 (2013).

18. Dubois, J. et al. Minimal-excitation states for electron quantum optics using levitons. Nature 502, 659-663 (2013).

19. Levitov, L. S., Lee, H. \& Lesovik, G. Electron counting statistics and coherent states of electric current. J. Math. Phys. 37, 4845-4886 (1996).

20. Lebedev, A. V., Lesovik, G. V. \& Blatter, G. Generating spin-entangled electron pairs in normal conductors using voltage pulses. Phys. Rev. B 72, 245314 (2005)

21. Keeling, J., Klich, I. \& Levitov, L. Minimal excitation states of electrons in one-dimensional wires. Phys. Rev. Lett. 97, 116403 (2006).

22. Beenakker, C. W. J., Emary, C., Kindermann, M. \& van Velsen, J. L. Proposal for production and detection of entangled electron-hole pairs in a degenerate electron gas. Phys. Rev. Lett. 91, 147901 (2003).

23. Beenakker, C. W. J., Titov, M. \& Trauzettel, B. Optimal spin-entangled electron-hole pair pump. Phys. Rev. Lett. 94, 186804 (2005)

24. Sherkunov, Y. B., d'Ambrumenil, N., Samuelsson, P. \& Büttiker, M. Optima pumping of orbital entanglement with single-particle emitters. Phys. Rev. B 85, 081108 (2012)

25. Yamamoto, M. et al. Electrical control of a solid-state flying qubit. Nature Nanotechnol. 7, 247-251 (2012).

26. Brantut, J. P. et al. Conduction of ultracold fermions through a mesoscopic channel. Science 337, 1069-1071 (2012)

27. Polycarpou, C., Cassemiro, K. N., Venturi, G., Zavatta, A. \& Bellini, M. Adaptive detection of arbitrarily shaped ultrashort quantum light states. Phys. Rev. Lett. 109 053602 (2012).

28. Dubois, J. et al. Integer and fractional charge Lorentzian voltage pulses analyzed in the framework of photon-assisted shot noise. Phys. Rev. B 88, 085301 (2013).

29. Pedersen, M. H. \& Büttiker, M. Scattering theory of photon-assisted electron transport. Phys. Rev. B 58, 12993 (1998)

30. Dasenbrook, D., Flindt, C. \& Büttiker, M. Floquet theory of electron waiting times in quantum-coherent conductors. Phys. Rev. Lett. 112, 146801 (2014).

31. Bocquillon, E. et al. Coherence and indistinguishability of single electrons emitted by independent sources. Science 339, 1054-1057 (2013).

Supplementary Information is available in the online version of the paper.

Acknowledgements We acknowledge the ERC Advanced Grant 228273 MeQuaNo and thank P. Jacques for technical help, P. Pari, P. Forget and M. de Combarieu for cryogenic support, and P. Degiovanni and C. Grenier for discussions improving the manuscript.

Author Contributions D.C.G. designed the project. T.J. and P.R. made the measurements and did the data analysis. B.R. contributed to the data analysis. P.R., T.J., B.R. and D.C.G. wrote the article. The sample was provided by Y.J. on wafer from A.C.

Author Information Reprints and permissions information is available at www.nature.com/reprints. The authors declare no competing financial interests. Readers are welcome to comment on the online version of the paper. Correspondence and requests for materials should be addressed to D.C.G. (christian.glattli@cea.fr). 